\title{
Therapeutic efficacy of erythropoietin alfa and erythropoietin beta in hemodialysis; a randomized controlled trial
}

\author{
Hamid-Reza Omrani $^{1}{ }^{\circledR}$, Sima Golmohhamadi $^{{ }^{*}}{ }^{(\mathbb{D}}$, Amir-Hossein Hashemian $^{2}$, Ali-Zaman Vaysmoradi ${ }^{1}$, \\ Roya Safari-Faramani
}

${ }^{1}$ Nephrology and Urology Research Center, Kermanshah University of Medical Sciences, Kermanshah, Iran

${ }^{2}$ Department of Biostatistics, Kermanshah University of Medical Sciences, Kermanshah, Iran

\section{A R T I C L E I N F O}

\section{Article Type:}

Original

\section{Article History:}

Received: 5 February 2018

Accepted: 25 July 2018

Published online: 13 August 2018

\section{Keywords:}

Erythropoietin alfa Erythropoietin beta

Kidney failure

Anemia

End-stage renal disease

Hemodialysis

\begin{abstract}
A B S T RAC T
Introduction: Anemia, as a common complication of end-stage renal disease (ESRD), usually develops due to erythropoietin deficiency. Recombinant human erythropoietins (rHEPOs) are indicated for the correction of renal anemia.

Objectives: We aimed to evaluate the efficacy of a new brand of erythropoietin named CinnaPoietin (erythropoietin beta) on hemoglobin levels.

Patients and Methods: This is a randomized double-blinded controlled trial. Ninety-six ESRD patients on hemodialysis recruited in the study, whose hemoglobin levels was less than $10 \mathrm{~g} / \mathrm{dL}$. They allocated to two groups. PDPoetin (erythropoietin alfa) 50-100 U/kg three times per week intravenously administrated to the control group and CinnaPoietin with exactly same regimen as like PDPoetin group administrated for the rest of the participants. The study duration was 3 months. We measured plasma hemoglobin monthly for 3 months. Results: We found, hemoglobin was increased across the time and it was statistically significant $(P<0.001)$, while there was no statistically significant differences between the groups $(P=0.712)$.

Conclusion: According to the result of the present study there is no statistical significant difference between these two brands of exogenous rHEPO in the case of increasing the hemoglobin concentration.
\end{abstract}

Implication for health policy/practice/research/medical education:

In a randomized controlled trial on 96 end-stage renal disease (ESRD) patients on hemodialysis, we found that administration of both brands (CinnaPoietin versus PDPoetin) of recombinant human erythropoietin is equally effective to increase the hemoglobin concentration in ESRD patients on hemodialysis.

Please cite this paper as: Omrani HR, Golmohhamadi S, Hashemian AH, Vaysmoradi AZ, Safari-Faramani RS. Therapeutic efficacy of erythropoietin alfa and erythropoietin beta in hemodialysis; a randomized controlled trial. J Renal Inj Prev. 2019;8(1):44-47. DOI: 10.15171/jrip.2019.09.

\section{Introduction}

Anemia is one of the most common problems in end-stage renal disease (ESRD) patients and its management is a matter of challenge for clinicians. The main reason is lack of erythropoietin hormone due to kidney damage. This exposes the patients to the risk of fatigue, breathlessness, blood transfusion, lower quality of life and some other problems (1,2). Erythropoiesis-stimulating agents (ESAs) have been available since the early 1980s and it was a great achievement in the management of anemia in dialysis patients (3-5). They are extremely important to maintain hemoglobin level in these patients as it has genteelly affect morbidity as well as quality of life. One of the most important advantages of ESAs is the prevention of blood transfusion and avoiding its adverse effects. The first recombinant human erythropoietin (rHEPO) produced was epoetin-alfa, followed by epoetin-beta, darbepoetinalfa, methoxy polyethylene glycol-epoetin beta, delta, omega and several related products $(3,4,6)$.

There are pieces of evidence on the efficacy and effectiveness of different types of rHEPOs. Solid evidence is available about the equivalence of beta and alfa rHEPOs in the case of efficacy $(1,7)$. Both of them are among the short acting erythropoietin stimulating agents and 
synthesized in Chinese hamster ovary $(\mathrm{CHO})$ cells with the same amino acid sequence. However there are some differences in their carbohydrate moieties as a result of different manufacturing processes $(8,9)$.

\section{Objectives}

Recently a new product of CinaGen Company named CinnaPoetin has been available at a lower cost. It is a rHEPOs beta. We aimed at assessing the efficacy of CinnaPoetin in ESRD patients who were on dialysis in the case of hemoglobin level increase.

\section{Patients and Methods}

\section{Study design}

This is a randomized double-blinded controlled trial (RCT) stated based on CONSORT (Figure 1). Ninety-six ESRD patients suffering from anemia (hemoglobin less than $10 \mathrm{mg} / \mathrm{dL}$ ) were enrolled in the study.

\section{Study population}

Participants were ESRD patients suffering from anemia (hemoglobin less than $10 \mathrm{mg} / \mathrm{dL}$ ) and they were on dialysis. Exclusion criteria were; suffering from uncontrolled hypertension, hospitalization during the last month or through the study period, blood transfusion within the preceding month or through the study period, iron deficiency (serum ferritin less than $200 \mu \mathrm{gram} / \mathrm{dl}$ ), serum parathormone more than $500 \mathrm{pg} / \mathrm{mL}$, C-reactive protein $>1+$, serum folic acid less than $3.1 \mathrm{pg} / \mathrm{mL}$, levels of vitamin B12 in serum less than $197 \mathrm{pg} / \mathrm{mL}$, using other stimulants such as nandrolone and other anabolic hormones, any unpleasant or adverse effects, presence of hemoglobinopathies and presence of anti-treatment resistant anemia such as myelodysplastic syndrome.

In the present study 103 patients were eligible out of 356. All patients referred to Imam Reza and Imam Khomeini hospitals.

The study conducted in Kermanshah, which is located in the west part of Iran. There are two main centers for providing dialysis services which are Imam Reza and Imam Khomeini hospitals. Both of these hospitals are educational and they cover all patients from different cities of the province.

First, the study introduced to the participants and the informed consents were signed, then we randomized patients into two groups. They were allocated to two intervention groups randomly via random number generation function in Excel. Additionally, both patients and personal in charge of the laboratory were blinded.

\section{Intervention}

Control group; we administrated $100 \mathrm{U} / \mathrm{kg}$, PDPoetin

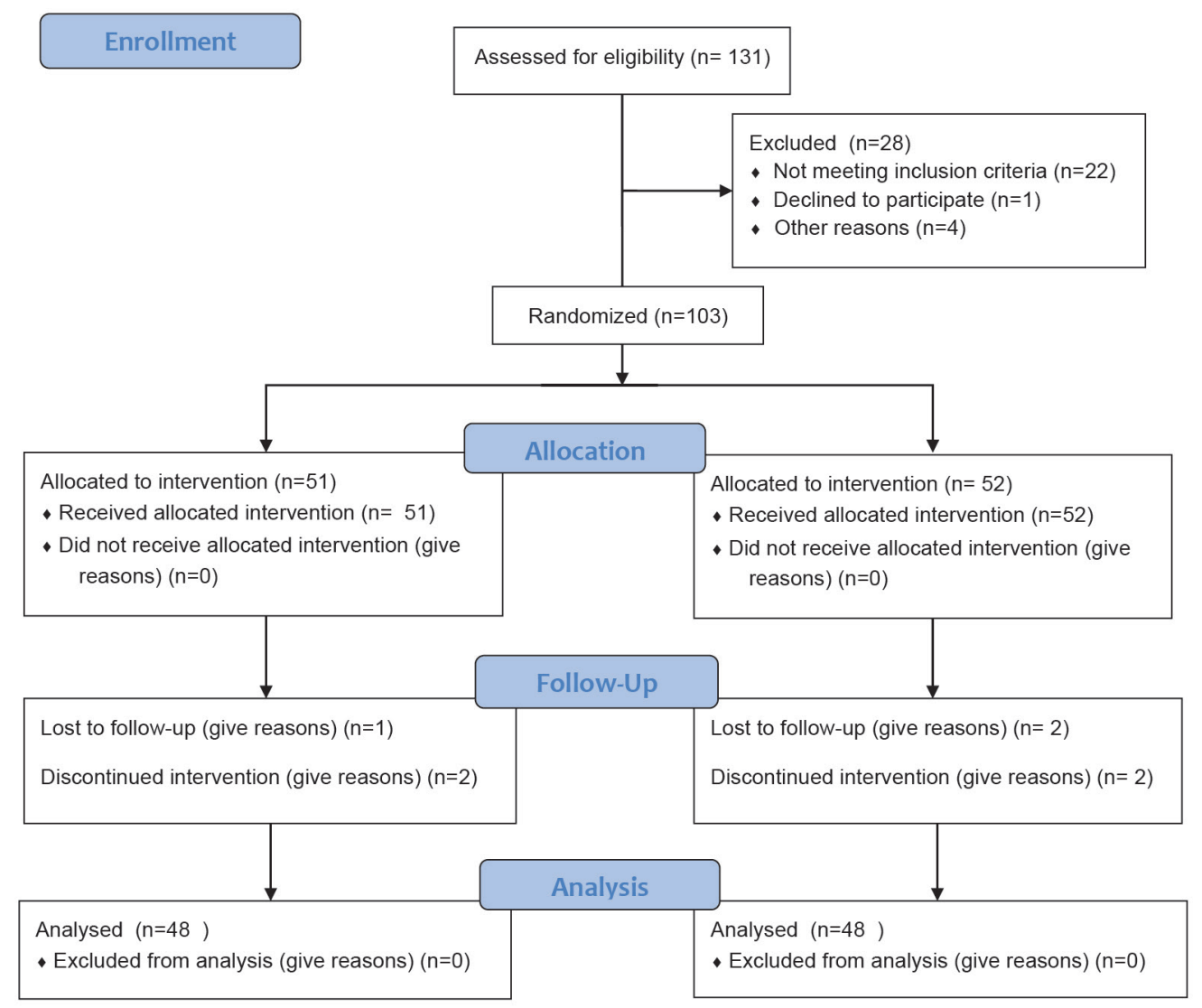

Figure 1. CONSORT flow diagram of the study. 
intravenously in three sessions across a week after the hemodialysis process in the control group. PDPoetin was manufactured by Pooyesh Daru Pharmaceutical Company in Iran.

Intervention group; $100 \mathrm{U} / \mathrm{kg}$ CinnaPoetin was administrated for the intervention group like the control group in time and frequency of injection. CinnaPoetin was manufactured by CinnaGen Pharmaceutical Company in Iran.

\section{Ethical issues}

The research followed the tenets of the Declaration of Helsinki. This study is a randomized controlled trial which registered in the Iranian Registry of Clinical Trials website (identifier: IRCT2013080812685N2; http:// www.irct.ir/trial/12701). This study was approved by the Ethics Committee of Kermanshah University of Medical Sciences (Ethics committee reference number: KUMS. REC.1394.144). From all subjects who participated in the investigation informed consent was obtained.

\section{Statistical analysis}

Quantitative data were reported by mean \pm standard deviation or mean $(95 \% \mathrm{CI})$ and qualitative data by percentage. Two groups were compared based on $\mathrm{U}$ Mann-Whitney and chi-square tests and were checked for assimilation by chi-square and independent t-test. The outcome variable was serum hemoglobin level. Repeated measure analysis was applied to assess the effect of the intervention across the time. The significance level was defined at 0.05 .

Results

Before randomization, 8 of the patients were excluded due to hospitalization (50\%) and death (50\%). In the case of baseline characteristics, there were no statistically significant differences between the groups (Table 1). In this study, we found that hemoglobin level has increased across the time and it was statistically significant $(P<0.001)$. The results are presented in Table 2 and also it is presented in Figure 2 schematically. There were no statistically significant differences between the groups $(P=0.712)$. It means that hemoglobin level in both of the groups increased equivalently. There were no statistically significant differences between sex $(P=0.243)$, age $(P=0.217)$, duration of hemodialysis $(P=0.25)$ or weight of the patients $(P=0.023)$ between the groups.

\section{Discussion}

This study was designed to assess the efficacy of CinnaPoetin as a new manufactured product by one of the Iranian pharmaceutical company. It is a rHEPOs beta. We intended to compare the efficacy of two brands of rHEPOs alfa and beta. According to the result of the present study, no statistical significant difference between these two brands of rHEPOs in the case of increasing in hemoglobin was detected. Hence, prescribing the new brand instead of previous PDPoetin will accompany with suitable results. The result of this study was consistent with the result of previous studies. Jumaa et al assessed the efficacy of 100 $\mu \mathrm{g}$ methoxy polyethylene glycol-epoetin beta every 2 weeks with 4000 IU epoetin alfa twice weekly to correct renal anemia in patients on hemodialysis (10). They did not report any statistically significant differences despite the positive and statistically significant effect of these agents on blood hemoglobin level after 3 months follow up. However, doses of the agents administrated in the present study were different from above mentioned study. Additionally Loughnanm et al did not detect any statistically significant differences between erythropoietin

Table 1. Baseline characteristics of patients participated in the study

\begin{tabular}{|c|c|c|c|}
\hline Variable & Control $(n=48)$ & Intervention $(n=48)$ & $P$ value \\
\hline Gender (female \%) & 35.42 & 52.08 & 0.1 \\
\hline Age, mean (SD) & $56.69(15.86)$ & 56.19 (13.99) & 0.4351 \\
\hline Weight, mean (SD) & $64.85(12.15)$ & $65.36(13.74)$ & 0.5763 \\
\hline Duration on hemodialysis (month) & $39.34(50.37)$ & $47.06(57.64)$ & 0.757 \\
\hline Baseline hemoglobin (g/dL) & $8.64(0.96)$ & $8.43(0.99)$ & 0.4379 \\
\hline Ferritin $(\mu \mathrm{g} / \mathrm{dL})$ & 474.35 (323.02) & $444.73(287.72)$ & 0.4379 \\
\hline PTH (pg/mL) & $182.17(127.58)$ & 214.04(139.56) & 0.8774 \\
\hline Vitamin B12 (pg/mL) & $729.77(554.26)$ & $695.45(564.51)$ & 0.3822 \\
\hline Folic acid (pg/mL) & $12.69(6.18)$ & $12.69(6.26)$ & 0.5262 \\
\hline
\end{tabular}

Table 2. Mean of hemoglobin level at each point of the study

\begin{tabular}{|c|c|c|c|c|}
\hline Time interval & PDPoetin, Mean $(95 \% \mathrm{Cl})$ & CinnaPoetin, Mean $(95 \% \mathrm{Cl})$ & Mean Difference $(95 \% \mathrm{Cl})$ & $P$ value \\
\hline Baseline & $8.47(8.19,8.74)$ & $8.43(8.15,8.72)$ & $0.03(-0.36,0.43)$ & 0.4379 \\
\hline Month 1 & $9.32(9.02,9.62)$ & $9.17(8.82,9.51)$ & $0.15(0.30,0.60)$ & 0.2556 \\
\hline Month 2 & $10.05(9.74,10.36)$ & $9.91(9.59,10.24)$ & $0.14(0.30,0.59)$ & 0.2642 \\
\hline Month 3 & $10.69(10.38,10.99)$ & $10.84(10.44,11.24)$ & $-0.15(-0.65,0.34)$ & 0.7312 \\
\hline
\end{tabular}




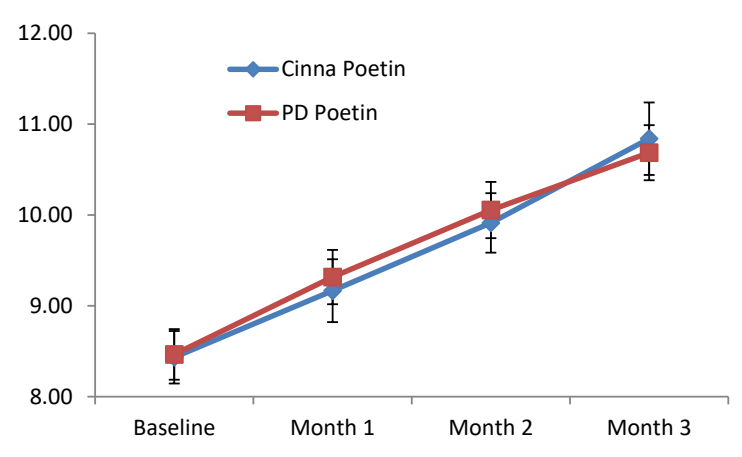

Figure 2. Trend of hemoglobin level in both intervention and control group.

alpha and beta in the case of increasing hemoglobin levels (11). However, in the case of needed dose they concluded that a lower dose of beta erythropoietin is needed to achieve the same hemoglobin level.

Up to our knowledge, it is the first study carried out to assess the efficacy of CinnaPoetin. We considered all the potential confounding factors. Vitamin B12 of serum, serum folic acid and intact PTH levels as well as the level of ferritin level are the influential factors in the response to erythropoietin stimulating agents. We checked these laboratory parameters at the beginning of the study (Table 1).

\section{Conclusion}

Based on the result of this study, administration of both brands of recombinant erythropoietin is effective to increase the hemoglobin level in ESRD patients on hemodialysis. Additionally, CinnaPoetin is equivalently effective to correct renal anemia in ESRD patients. This evidence may be helpful for clinicians to choose the best option for renal anemia management.

\section{Study limitations}

Great deals of evidence on the effectiveness of rHEPO agents in correcting renal anemia are available. However, to our knowledge, there is no identical study to assess the effectiveness of the CinnaPoetin in renal disease patients. Designing and carrying out large, multicenter randomized controlled trials to assess its efficacy is recommended for the prospective researches. Furthermore we administrated both of the agents intravenously. As the route of administration may affect its half-life as well as its efficacy, carrying out studies including different types of administration, subcutaneously or intravenously is recommended. Besides improving post marketing surveillance systems to monitor its probable side effects may shed light on decision making about the best practice.
Authors' contribution

HRO and SG designed the study, observed accuracy and validity of the study. AHH and AZV collected the data and follow the study. RS-F analyzed the data. SG wrote the paper. All authors edited and revised the final manuscript.

\section{Conflicts of interest}

The authors declare that the preference of one brand over other ones does not imply the bias of authors and it is only the result of authors' experiment.

\section{Funding/Support}

This article resulted from the research project of 95312 funded by deputy of research, Kermanshah University of Medical Sciences.

\section{References}

1. Thilaka GK, Kumar SV. A review on pharmacological use of recombinant human erythropoietin in renal and nonrenal anemia and other potential applications in clinical practice. Apollo Med. 2016;13:80-85.

2. Hörl WH. Anaemia management and mortality risk in chronic kidney disease. Nat Rev Nephrol. 2013;9:291-301. doi: 10.1038/nrneph.2013.21.

3. Ansari I, Sheikh A, Ahmed SS, Jabbar Q, Ali S. Management of Anemia and other Hematologic Derangements in Patients with Chronic Kidney Disease. Arab J Nephrol Transplant. 2014;7:13-9.

4. Deicher R, Hörl WH. Differentiating factors between erythropoiesis-stimulating agents: a guide to selection for anaemia of chronic kidney disease. Drugs. 2004;64:499509.

5. Ören B, Zengin N. The effect of anemia on quality of life and self-care agency in Turkey hemodialysis patients. Open J Nurs. 2016;6:443-448.

6. Malyszko J, Malyszko JS. Emerging drugs for the treatment of kidney disease-induced anemia. Expert Opin Emerg Drugs. 2016;21:315-30. doi: 10.1080/14728214.2016.1220537.

7. Locatelli F, Del Vecchio L. Erythropoiesis-stimulating agents in renal medicine. Oncologist. 2011;16:19-24. doi: 10.1634/theoncologist.2011-S3-19.

8. Hörl WH. Differentiating factors between erythropoiesisstimulating agents: an update to selection for anaemia of chronic kidney disease. Drugs. 2013;73:117-30. doi: 10.1007/s40265-012-0002-2.

9. Wu MJ. Which ESAs You Will Choose for Dialysis Patients? Acta Nephrologica. 2012; 26:134-136. doi: 10.6221/ AN.2012026.

10. Jumaa AK, Al Temimi HMA, Alsabbagh MS, Rasheed JI. Efficacy and safety of methoxy polyethylene glycol epoetin - beta versus epoetin alpha for treatment of chronic renal anemia in hemodialysis patient. Am J Pharmacol Sci. 2015;3:87-93. doi: 10.12691/ajps-3-4-1

11. Loughnan A, Ali GR, Abeygunasekara SC. Comparison of the therapeutic efficacy of epoetin beta and epoetin alfa in maintenance phase hemodialysis patients. Ren Fail. 2011;33:373-5. doi: 10.3109/0886022X.2011.559675.

Copyright $\odot 2019$ The Author(s); Published by Nickan Research Institute. This is an open-access article distributed under the terms of the Creative Commons Attribution License (http://creativecommons.org/licenses/by/4.0), which permits unrestricted use, distribution, and reproduction in any medium, provided the original work is properly cited. 